\title{
SISTEMA DE INFORMAÇÃO GEOGRÁFICA PARA MAPEAMENTO DA RENDA LÍQUIDA APLICADO NO PLANEJAMENTO DA AGRICULTURA IRRIGADA ${ }^{1}$
}

\author{
WILSON A. SILVA ${ }^{2}$, DANIEL F. CARVALHO ${ }^{3}$, CARLOS A. A. VARELLA ${ }^{4}$, \\ MARCOS B. CEDDIA ${ }^{5}$
}

\begin{abstract}
RESUMO: O objetivo deste trabalho foi desenvolver um algoritmo na linguagem computacional MATLAB para aplicações em sistemas de informações geográficas, visando ao mapeamento da renda líquida maximizada de cultivos irrigados. O estudo foi desenvolvido para as culturas do maracujá, da cana-de-açúcar, do abacaxi e do mamão, em área de aproximadamente 2.500 ha, localizada no município de Campos dos Goytacazes, norte do Estado do Rio de Janeiro. Os dados de entrada do algoritmo foram informações edafoclimáticas, funções de resposta das culturas à água, dados de localização geográfica da área e índices econômicos referentes ao custo do processo produtivo. Os resultados permitiram concluir que o algoritmo desenvolvido se mostrou eficiente para o mapeamento da renda líquida de cultivos irrigados, sendo capaz de localizar áreas que apresentam maiores retornos econômicos.
\end{abstract}

PALAVRAS-CHAVE: SIG, agricultura irrigada, funções de produção.

\section{ALGORITHM TO MAPPING NET INCOME APPLIED IN IRRIGATED AGRICULTURE PLANNING}

\begin{abstract}
The objective of this work was to develop an algorithm in MATLAB computational language to be applied in geographical information systems to map net income irrigated crops to plan irrigated agriculture. The study was developed for the crops of passion fruit plant, sugarcane, pineapple and papaya, in an area of approximately 2,500 ha, at Campos dos Goytacazes, located at north of the State of Rio de Janeiro, Brazil. The algorithm input data were: information about soil, climate, crop water response functions, geographical location and economical cost indexes of the productive process. The results allowed concluding that developed algorithm was efficient to map net income of irrigated crops, been able to locate areas that present larger economical net income.
\end{abstract}

KEYWORDS: GIS, irrigated agriculture, production functions.

\section{INTRODUÇÃO}

No planejamento de atividades agrícolas, o uso dos sistemas de informações geográficas (SIG) tem-se mostrado como importante ferramenta, possibilitando criar de forma prática, rápida e menos onerosa, bancos de dados e documentos cartográficos com as mais diversas informações que servirão de base para o adequado planejamento de cultivos irrigados (SILVA, 2003).

O termo geoprocessamento denota a disciplina do conhecimento que utiliza técnicas matemáticas e computacionais para o tratamento de informações geográficas (CAMARA \& MEDEIROS, 1998). De acordo com ASSAD \& SANO (1998), os SIGs são considerados os instrumentos computacionais do geoprocessamento, podendo ser conceituados também como um conjunto de ferramentas que permite a coleta, o armazenamento, a recuperação, a transformação e a apresentação de dados do mundo real para determinados objetivos específicos, que geralmente são o apoio à tomada de decisões.

\footnotetext{
${ }^{1}$ Extraído da Tese de Doutorado do primeiro autor.

${ }^{2}$ Eng $^{\mathrm{o}}$ Agrônomo, Prof. Doutor, UEMA - Câmpus III, Imperatriz - MA, wilsonufrrj@yahoo.com.br

${ }^{3}$ Eng ${ }^{\mathrm{O}}$ Agrícola, Prof. Doutor, Departamento de Engenharia/Instituto de Tecnologia/UFRRJ, BR 465, km 7, Seropédica - RJ, carvalho@ufrrj.br, Bolsista do CNPq.

${ }^{4}$ Engo Agrônomo, Prof. Doutor, Departamento de Engenharia/Instituto de Tecnologia/UFRRJ, BR 465, km 7, Seropédica - RJ, varella@ufrrj.br

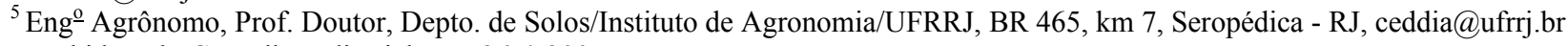
Recebido pelo Conselho Editorial em: 26-4-2007
}

Aprovado pelo Conselho Editorial em: 21-1-2008 
Diversos trabalhos têm sido realizados utilizando o SIG para diferentes aplicações e objetivos. RODRIGUEZ \& LOPEZ (2000) comprovaram a aplicabilidade do SIG no planejamento de sistemas de irrigação e drenagem em cultivos de arroz irrigado, produzindo mapas temáticos que auxiliaram na modernização dos sistemas de irrigação em Cuba. FARIA et al. (2002) estudaram a economia de água e energia em projetos de irrigação suplementar no Estado de Minas Gerais, para a cultura do milho (Zea mays L.). Os autores observaram, por meio de documentos cartográficos gerados pelo SIG IDRISI, diferenças entre a demanda de irrigação total e a suplementar, e puderam estudar a conveniência de incluir ou não a contribuição das precipitações em um projeto de irrigação.

CARVALHO et al. (1998) realizaram estudos utilizando o SIG IDRISI 3.0 para estimar a demanda máxima de irrigação e duração do ciclo da cultura do milho (Zea mays L.) na bacia do rio Verde Grande, em Minas Gerais, e concluíram que a metodologia utilizada permitiu a identificação das informações estudadas em qualquer ponto dentro da bacia, sendo indicada para outras regiões, principalmente aquelas que apresentam baixa disponibilidade de água para irrigação.

Utilizando os sistemas de informações geográficas no estudo de disponibilidade climática no Estado do Rio de Janeiro, visando à elaboração de projetos de irrigação, CARVALHO et al. (2001) concluíram que, utilizando-se do SIG, é possível identificar áreas e épocas do ano mais viáveis climaticamente à atividade agrícola. CARVALHO et al. (2002) compararam diferentes metodologias de estimativa da evapotranspiração de referência e sua influência na demanda máxima de irrigação para o milho (Zea mays L.), no Estado do Rio de Janeiro, utilizando-se do SIG, e verificaram a importância dessa ferramenta na produção de documentos cartográficos.

CARVALHO et al. (2005) utilizaram o SIG IDRISI em áreas de assentamentos rurais com o objetivo de indicar áreas agroeconomicamente mais aptas para a implantação da cultura do coqueiro irrigado, utilizando mapas digitais de solo e planialtimetria, além de mapas temáticos com informações de drenagem, estradas e limite dos lotes. Os autores concluíram que a metodologia mostrou-se eficaz para a implantação de culturas irrigadas, sendo facilmente aplicável para outras culturas e áreas de estudo.

CEDDIA (2000) comprovou a adequação da utilização de SIG no estudo de zoneamento agroambiental e indicadores de sustentabilidade como subsídio ao planejamento agrícola do município de Paty do Alferes - RJ. O autor concluiu que o uso de ferramentas de análise de pertinência do SIG ARC/INFO permitiu o ganho de conhecimento sobre o município, no que diz respeito às inter-relações dos componentes do meio físico e as incongruências entre uso e recomendação de uso do solo.

O planejamento da agricultura irrigada torna-se ainda mais necessário, pois além da importância fisiológica que a água e os nutrientes desempenham na produção vegetal, suas aplicações, no momento oportuno e nas quantidades necessárias, são de fundamental importância para que se consiga obter o rendimento líquido máximo (SILVA, 2007).

Com base no exposto, percebe-se o potencial de aplicação do SIG no planejamento da agricultura. Tendo em vista a importância do uso dessa ferramenta, este trabalho teve como objetivo desenvolver um algoritmo computacional para aplicações em SIG que possibilite a espacialização da renda líquida maximizada e avaliar a sua aplicação no planejamento da agricultura irrigada na região norte do Estado do Rio de Janeiro.

\section{MATERIAL E MÉTODOS}

\section{Caracterização da área de estudo}

O estudo foi realizado para uma área de aproximadamente 2.500 ha pertencente ao PROJIR (Projeto de Irrigação e Drenagem da Cana-de-Açúcar na Região Norte Fluminense), executado em 1982 pelo Instituto do Açúcar e do Álcool, por meio do PLANALSUCAR. Essa área localiza-se no município de Campos dos Goytacazes, região norte do Estado do Rio de Janeiro, e faz parte do 
ambiente dos tabuleiros costeiros, onde predomina o relevo plano a suave ondulado. As classes de solo de maior expressão na área estudada são os Latossolos, os Cambissolos e os Neossolos flúvicos. A área está inserida na microbacia hidrográfica do rio Muriaé, entre as coordenadas geográficas de $21^{\circ} 37^{\prime} 30^{\prime \prime}$ e $21^{\circ} 39^{\prime} 54^{\prime}$ 'sul e $41^{\circ} 22^{\prime} 30^{\prime \prime}$ e $41^{\circ} 26^{\prime} 15^{\prime}$ 'oeste.

\section{Construção do algoritmo computacional}

$\mathrm{O}$ algoritmo foi desenvolvido na linguagem computacional MATLAB. Os dados de entrada foram informações edafoclimáticas, funções de resposta das culturas à água, dados de localização geográfica da área e índices econômicos referentes ao custo do processo produtivo. Com base nesses dados, o algoritmo gerou um valor de renda líquida maximizada para cada célula do mapa. Dessa maneira, a renda líquida maximizada foi subdividida em quatro classes diferentes para cada uma das culturas estudadas. Na Figura 1, ilustram-se as etapas do algoritmo computacional desenvolvido.

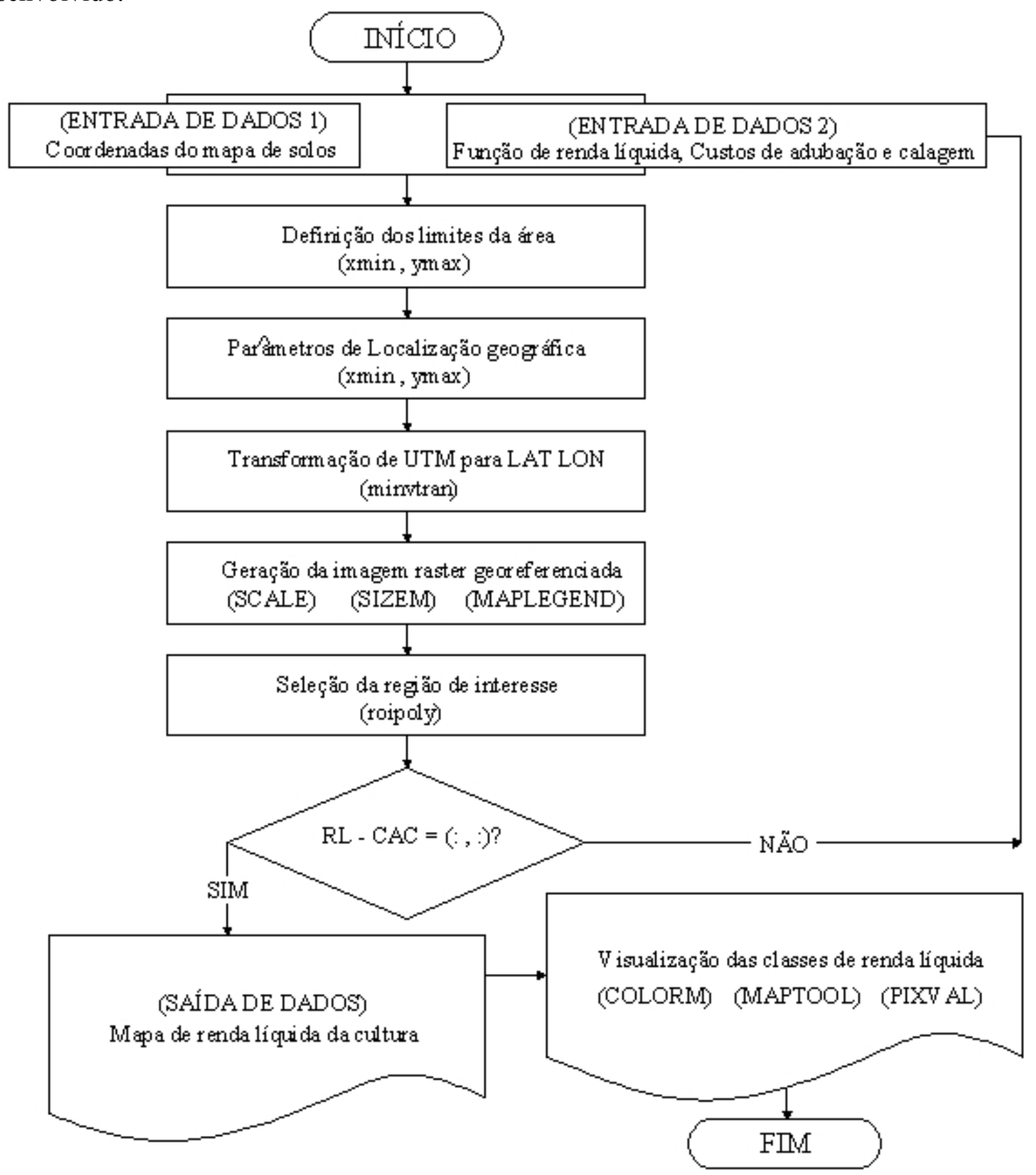

FIGURA 1. Etapas do algoritmo computacional em desenvolvimento. Step of computational algorithm development. 


\section{Geração e georreferenciamento da imagem raster}

Nessa etapa, as informações contidas no PROJIR, como mapas de solo e planialtimétrico (escala 1:10.000), foram convertidas do formato analógico para o formato digital matricial e, posteriormente, convertidos para o formato vetorial, utilizando-se do módulo CAD do software TOPO EVN 5.0 para Windows. A partir do mapa vetorizado de solos (Figura 2), foram selecionadas as coordenadas de cada unidade de mapeamento que apresentavam características físicas, químicas e mineralógicas similares às utilizadas para a obtenção das funções de respostas à água das culturas estudadas.

As coordenadas UTM (Universal Transverse Mercator) de cada unidade de mapeamento foram armazenadas em arquivos '*.txt' e inseridas no algoritmo por meio dos comandos $S A V E$ e $\angle O A D$ do pacote MATLAB. O limite da área em estudo foi definido em função da diferença entre os valores extremos das coordenadas $\mathrm{X}$ e $\mathrm{Y}\left(\mathrm{x}_{\min }\right.$ e $\left.\mathrm{y}_{\max }\right)$. A estrutura de localização geográfica do mapa gerado foi definida com base na projeção UTM para o fuso $23 \mathrm{~S}$ e geóide de referência Clarke 66. Como os parâmetros do sistema geodésico utilizado no Brasil (SAD 69) não são os mesmos do geóide Clarke 66, foram feitas correções a fim de utilizar os mesmos parâmetros do sistema geodésico brasileiro. Após essa etapa, foi realizada a conversão das coordenadas UTM para coordenadas geográficas (SILVA, 2007).
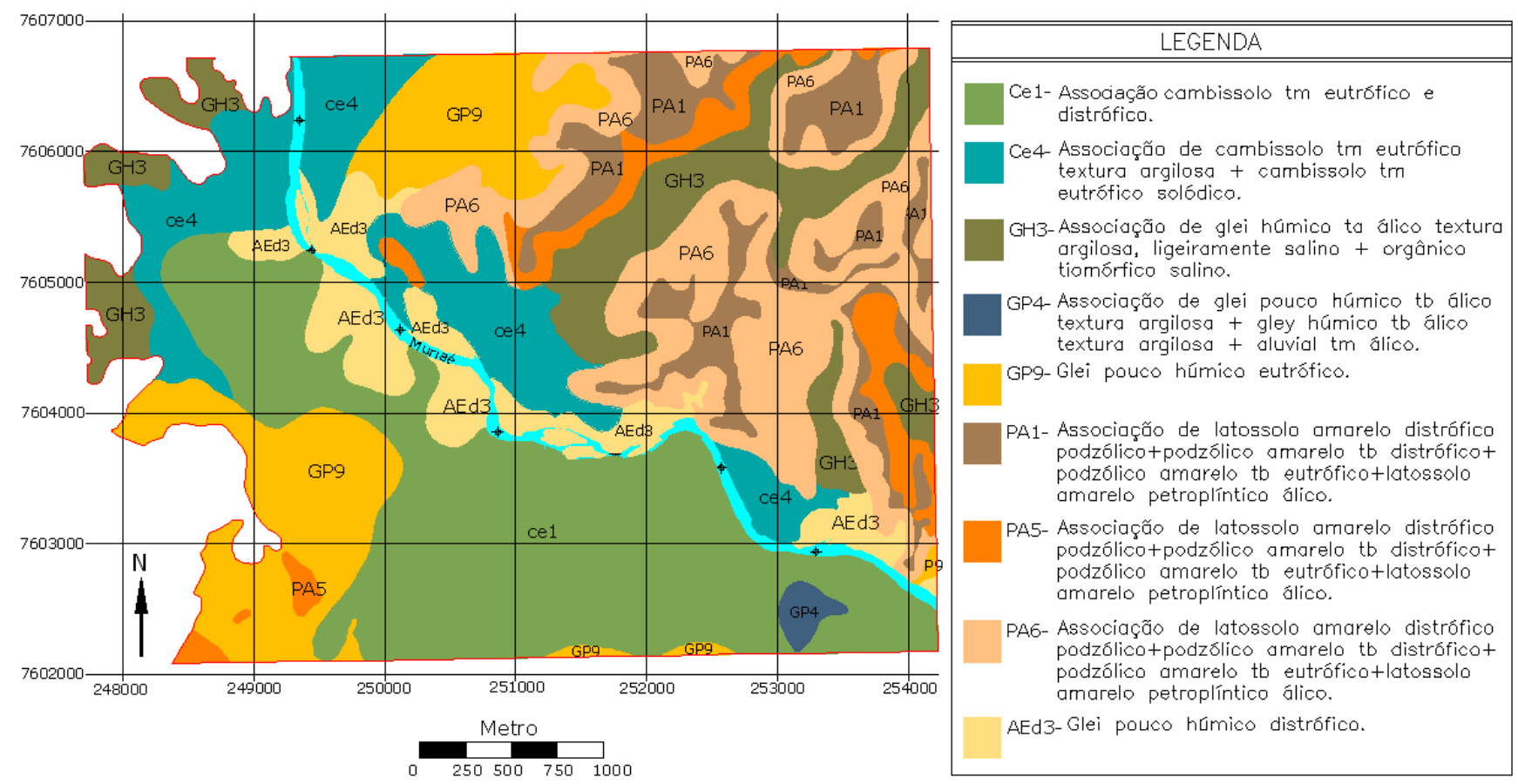

FIGURA 2. Mapa de solos da área estudada. Soil map of the study site.

O processo de geração da imagem raster foi realizado em três etapas: definição da resolução espacial da imagem; definição das dimensões do arquivo matricial, e georreferenciamento da imagem.

Nesse estudo, foi adotada resolução espacial de $10 \mathrm{~m}$, ou seja, cada pixel da imagem raster representou uma área de $10 \mathrm{~m}$ x $10 \mathrm{~m}$ do mundo real. Como o sistema de unidades de projeção do mapa gerado trabalha em graus decimais, foi determinado um fator de escala (scale) que mostrou o quanto cada pixel da matriz da imagem representa no mundo real.

Em função da resolução espacial adotada, foi criado um retângulo envolvente da área estudada, com dimensões de $6.675 \mathrm{~m}$ x $4.650 \mathrm{~m}$, que foi dividido pela resolução espacial adotada, gerando matriz de 464 linhas por 666 colunas. A implementação dessa etapa no MATLAB 6.0 foi realizada utilizando-se, como parâmetros de entrada, dos valores extremos ou limites de latitude e longitude da área e do fator de escala SCALE (limlat, limlong, scale). 
O georreferenciamento da imagem foi realizado utilizando a função MAPLEGEND, que definiu a localização geográfica e o tamanho da célula da matriz do mapa, utilizando como parâmetros de entrada o fator de escala e os maiores valores de latitude na direção norte e oeste [scale maxlat minlon]. Cumprida essa etapa, as regiões de interesse dentro da matriz do mapa foram selecionadas utilizando a função ROIPOLY. Essa função gerou uma imagem binária com valor um (1) dentro da área de interesse e zero (0) do lado de fora. As regiões consideradas de interesse foram as próprias unidades de mapeamento, que posteriormente foram multiplicadas pelo valor da renda líquida de cada cultura.

\section{Maximização da renda líquida}

Tendo em vista o fato de não existirem pontos específicos de captação na área estudada, não foram consideradas, no cálculo da renda líquida, as despesas com tubulação e aquisição de motobomba, conforme SILVA et al. (2005), além dos gastos de energia elétrica.

A renda maximizada de cada cultura foi calculada, conforme a eq.(1), considerando, para isso, estudo de maximização da renda decorrente dos cultivos irrigados das culturas do maracujá, da cana-de-açúcar, do abacaxi e do mamão, sujeitas às restrições na disponibilidade de água e área para cultivo. Na eq.(1), não foram contemplados os custos de adubação e de calagem, uma vez que os mesmos foram trabalhados de maneira georreferenciada para cada unidade de mapeamento e, posteriormente, incorporados na análise.

$$
\mathrm{R}_{\max }=\left(\text { prec } \mathrm{p}_{\max }\right)-\text { cust }-\left(\text { cust }_{\mathrm{w}} \mathrm{w}_{\max }\right)
$$

em que,

$\mathrm{R}_{\max }$ - renda máxima da cultura, $\mathrm{R} \$ \mathrm{ha}^{-1}$;

prec - preço de venda da tonelada do produto, $\mathrm{R} \$ \mathrm{t}^{-1}$;

$\mathrm{p}_{\max }$ - produtividade máxima em função da lâmina de água aplicada, $\mathrm{t} \mathrm{ha}^{-1}$;

cust - custo de produção desconsiderando os gastos com água, adubação e calagem, $\mathrm{R} \$ \mathrm{ha}^{-1}$;

cust $_{\mathrm{w}}$ - custo da água, $\mathrm{R} \$ \mathrm{~mm}^{-1}$, e

$\mathrm{W}_{\max }$ - lâmina ótima associada ao máximo retorno econômico, $\mathrm{mm}$.

O modelo matemático de otimização utilizado foi o Langrangiano Aumentado, conforme JUSSIANE (2004), e foi desenvolvido considerando a época de cultivo das diferentes culturas, sendo a disponibilidade máxima de água para cada mês calculada em função da vazão possível de ser outorgada na seção do rio Muriaé, localizada na área de estudo (SILVA, 2007). Como valores de área máxima cultivada por cultura, foram considerados aqueles proporcionais às áreas ocupadas com as respectivas culturas no município de Campos dos Goytacazes.

$\mathrm{O}$ valor de $\mathrm{R}_{\max }$ constituiu o atributo de cada célula do mapa gerado e para o seu cálculo foram utilizadas informações relativas ao custo de produção das culturas apresentado na Tabela 1. Para o cálculo de $p_{\max }$, foram utilizadas as funções de resposta apresentadas na Tabela 2, as quais foram obtidas por SOUSA (1997) (cana-de-açúcar), REGO FILHO (2002) (abacaxi), MARTINS (1998) (maracujá) e ALMEIDA (2000) (mamão), em experimentos de campo, para as condições edafoclimáticas semelhantes às da área estudada.

A componente $\mathrm{w}_{\max }$, na eq.(1), considera a demanda mensal de água das culturas e foi estimada com base no balanço hídrico realizado em função da precipitação provável e da evapotranspiração potencial das culturas. Para isso, foram utilizados os dados climáticos diários da estação de Campos dos Goytacazes, cadastrada no INMET com o código 83698. O cust $_{\mathrm{w}}$ foi calculado considerando a metodologia de cobrança utilizada pelo Comitê Executivo para Integração da Bacia do Rio Paraíba do Sul - CEIVAP, que adota preço público unitário para irrigação de R\$ 0,0005 por $\mathrm{m}^{3}$. Os custos de adubação e de calagem foram obtidos conforme critérios apresentados por De-POLLI et al. (1988), utilizando dados de análises químicas de amostras representativas dos solos em cada unidade de mapeamento da área em estudo (Tabela 3 ). 
TABELA 1. Custos de produção e preços de venda utilizados no desenvolvimento do algoritmo. Production costs and selling price used in the algorithm development.

\begin{tabular}{ccc}
\hline Cultura & Custo de Produção $\left(\mathrm{R} \$\right.$ ha $\left.^{-1}\right)$ & Preço do Produto $\left(\mathrm{R} \$\right.$ t $\left.^{-1}\right)$ \\
\hline Cana-de-açúcar & $8.171,37$ & 56,00 \\
Abacaxi & $8.571,17$ & 230,00 \\
Mamão & $10.191,60$ & 320,00 \\
Maracujá & $11.784,20$ & 340,00 \\
\hline
\end{tabular}

Fonte: AGRIANUAL (2004).

TABELA 2. Funções de resposta à água para as culturas estudadas. Water response function of the crops studied.

\begin{tabular}{lcc}
\hline \multicolumn{1}{c}{ Cultura } & \multicolumn{1}{c}{ Função de Resposta } & $\mathrm{r}^{2}$ \\
\hline Cana-de-açúcar & $\mathrm{Y}=-500,84+0,837 \mathrm{~W}-0,00027 \mathrm{~W}^{2}$ & 0,89 \\
Abacaxi & $\mathrm{Y}=-506,687+0,905709 \mathrm{~W}-0,000370675 \mathrm{~W}^{2}$ & 0,98 \\
Maracujá & $\mathrm{Y}=2,3931+0,049058 \mathrm{~W}-0,0000183 \mathrm{~W}^{2}$ & 0,68 \\
Mamão & $\mathrm{Y}=-88,13+0,098092 \mathrm{~W}-0,00001725 \mathrm{~W}^{2}$ & 0,92 \\
\hline
\end{tabular}

$\mathrm{Y}=$ produtividade, $\mathrm{t} \mathrm{ha}^{-1} ; \mathrm{W}=$ lâmina de água total do ciclo, $\mathrm{mm}$.

TABELA 3. Custos de adubação e calagem utilizados no desenvolvimento do algoritmo. Soil liming and fertilization costs used to algorithm development.

\begin{tabular}{|c|c|c|c|c|}
\hline \multirow{2}{*}{$\begin{array}{l}\text { Unidade de } \\
\text { Mapeamento }\end{array}$} & \multicolumn{4}{|c|}{ Custos de Adubação e de Calagem $\left(\mathrm{R} \$\right.$ ha $\left.^{-1}\right)$} \\
\hline & Maracujá & Cana-de-açúcar & Abacaxi & Mamão \\
\hline $\mathrm{Ce} 4$ & 408,00 & 185,00 & $1.400,00$ & 549,00 \\
\hline $\mathrm{Ce} 1$ & 432,00 & 196,00 & $1.480,00$ & 564,00 \\
\hline PA1 & 469,00 & 222,00 & 883,00 & 597,00 \\
\hline Aed3 & 545,00 & 190,00 & $1.553,00$ & 695,00 \\
\hline
\end{tabular}

A renda líquida maximizada ( $R L_{\max }$ ) foi obtida na forma de mapa, subtraindo o valor de $\mathrm{R}_{\max }$, obtido a partir da eq.(1), do custo de adubação e de calagem para cada cultura e para cada unidade de mapeamento. A apresentação de todos os mapas correspondentes aos limites de cada unidade em uma única imagem foi realizada somando-se os mapas individuais de cada unidade de mapeamento.

Para visualizar as classes de renda no mapa final, foi utilizada a ferramenta interativa COLORM, que permite a apresentação colorida da matriz do mapa, possibilitando separar, por meio de uma paleta de cores, as classes de valores da matriz do mapa gerado. A fim de permitir a visualização do eixo de projeção, criado utilizando a função MESHM na imagem do mapa final gerado, foi utilizada a ferramenta interativa MAPTOOL, que permite a visualização dos parâmetros de localização geográfica na imagem gerada e do eixo em função do sistema de projeção.

\section{RESULTADOS E DISCUSSÃO}

Os resultados encontrados pelo algoritmo computacional estão apresentados na Tabela 4 e nas Figuras 3 a 6. É possível notar que o algoritmo foi capaz de mapear as classes de renda liquida das culturas estudadas e representar essas classes em diferentes paletas de cores em uma interface gráfica. A obtenção das classes de renda líquida foi influenciada pelas diferenças entre características químicas e físico-hídricas apresentadas pelos solos da área em estudo.

As classes com melhores níveis de fertilidade natural e maior capacidade de retenção de água contribuíram para a maior renda líquida. Foi possível perceber, também, que as culturas do abacaxi e do mamão apresentaram os maiores valores de renda líquida na área estudada. As informações geradas pelo algoritmo podem auxiliar na seleção de padrões de cultivo que propiciem maiores rendimentos econômicos quando da implantação dessas culturas na região. 
TABELA 4. Valores de renda líquida obtidos pelo algoritmo nos diferentes tipos de solos. Values of net income obtained by algorithm in different soil types.

\begin{tabular}{clccl}
\hline \multirow{2}{*}{ Unidade de Mapeamento } & \multicolumn{4}{c}{ Renda Líquida $\left(\mathrm{R} \$\right.$ ha $\left.^{-1}\right)$} \\
\cline { 2 - 5 } & Maracujá & Cana-de-Açúcar & Abacaxi & Mamão \\
\hline Ce1 & $2.492,00$ & 345,00 & 32.873 & 6.218 \\
Ce4 & $2.516,00^{* *}$ & $356,00^{* *}$ & 32.953 & $6.233^{* *}$ \\
PA1 & $2.455,00$ & $319,00 *$ & $33.470^{* *}$ & 6.185 \\
Aed3 & $2.379,00 *$ & 351,00 & $32.800 *$ & $6.087^{*}$ \\
\hline
\end{tabular}

* menor valor de renda líquida da cultura; ** maior valor de renda líquida da cultura.

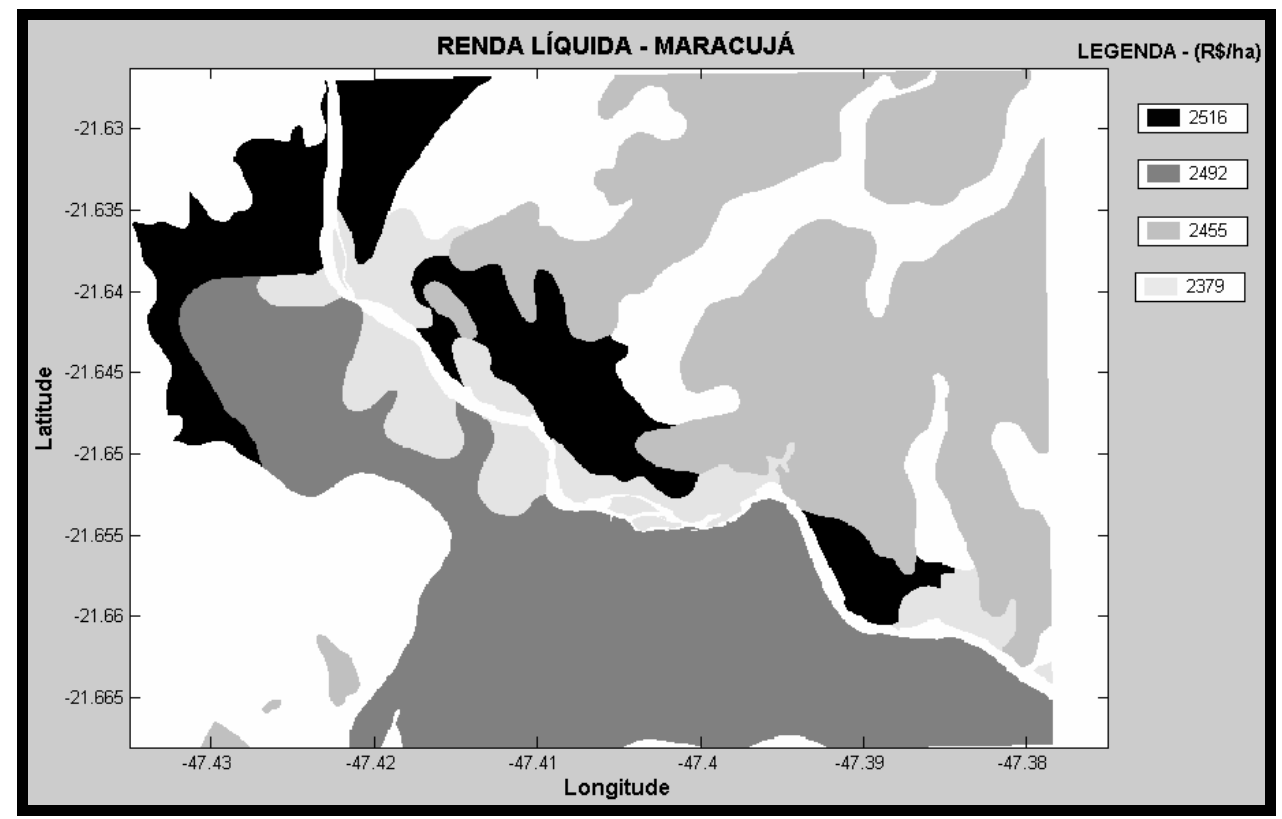

FIGURA 3. Tela de saída do algoritmo, mostrando a renda líquida da cultura do maracujá. Algorithm output screen showing the income obtained planting passion fruit.

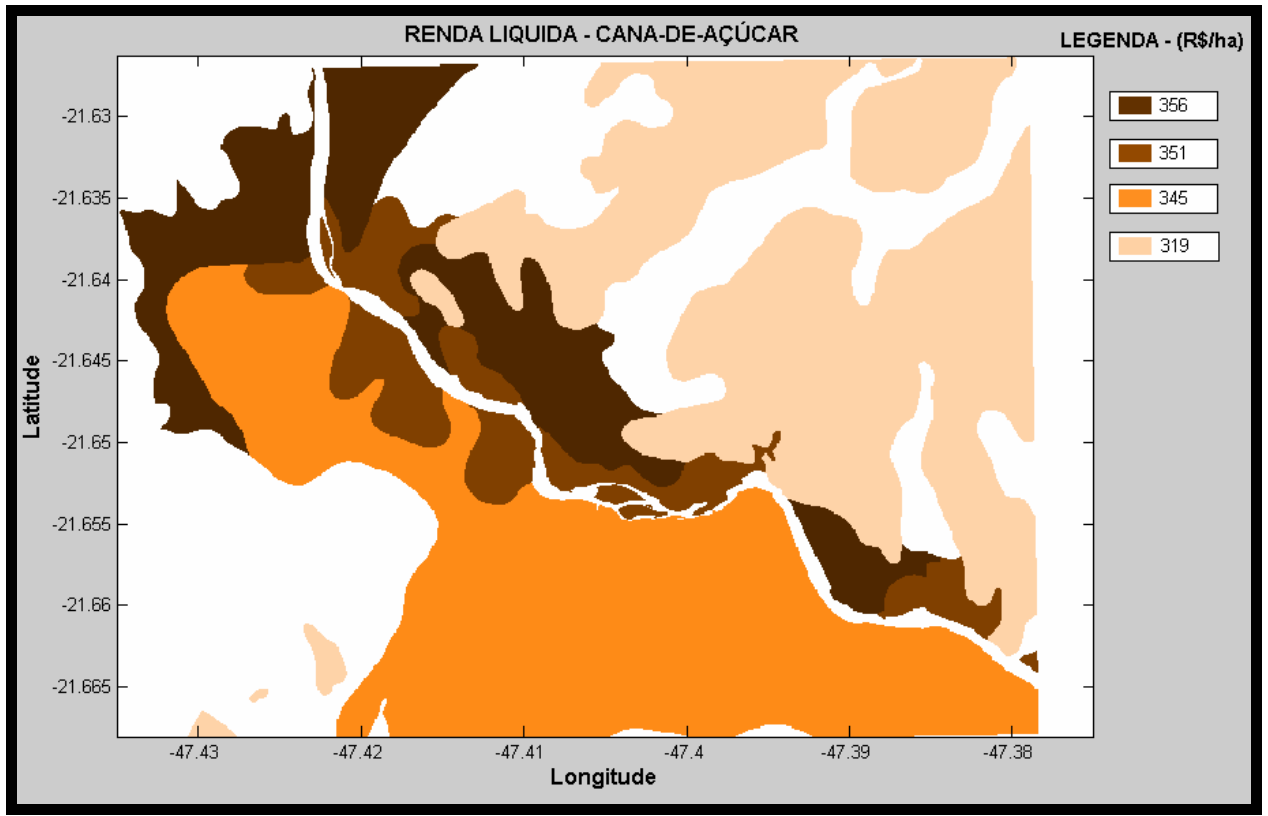

FIGURA 4. Tela de saída do algoritmo, mostrando a renda líquida da cultura da cana-de-açúcar. Algorithm output screen showing the income obtained planting sugar cane. 


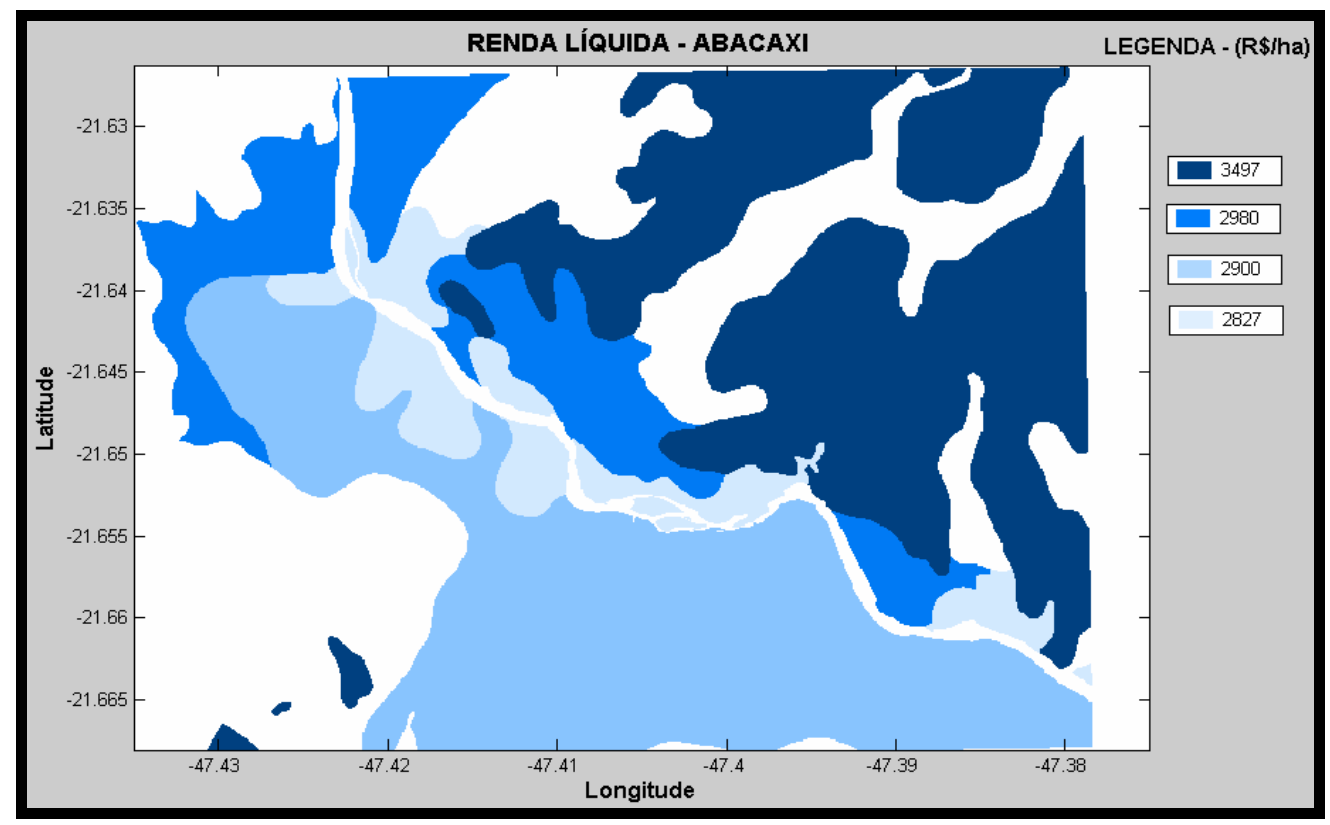

FIGURA 5. Tela de saída do algoritmo, mostrando a renda líquida da cultura do abacaxi. Algorithm output screen showing the income obtained planting pineapple.

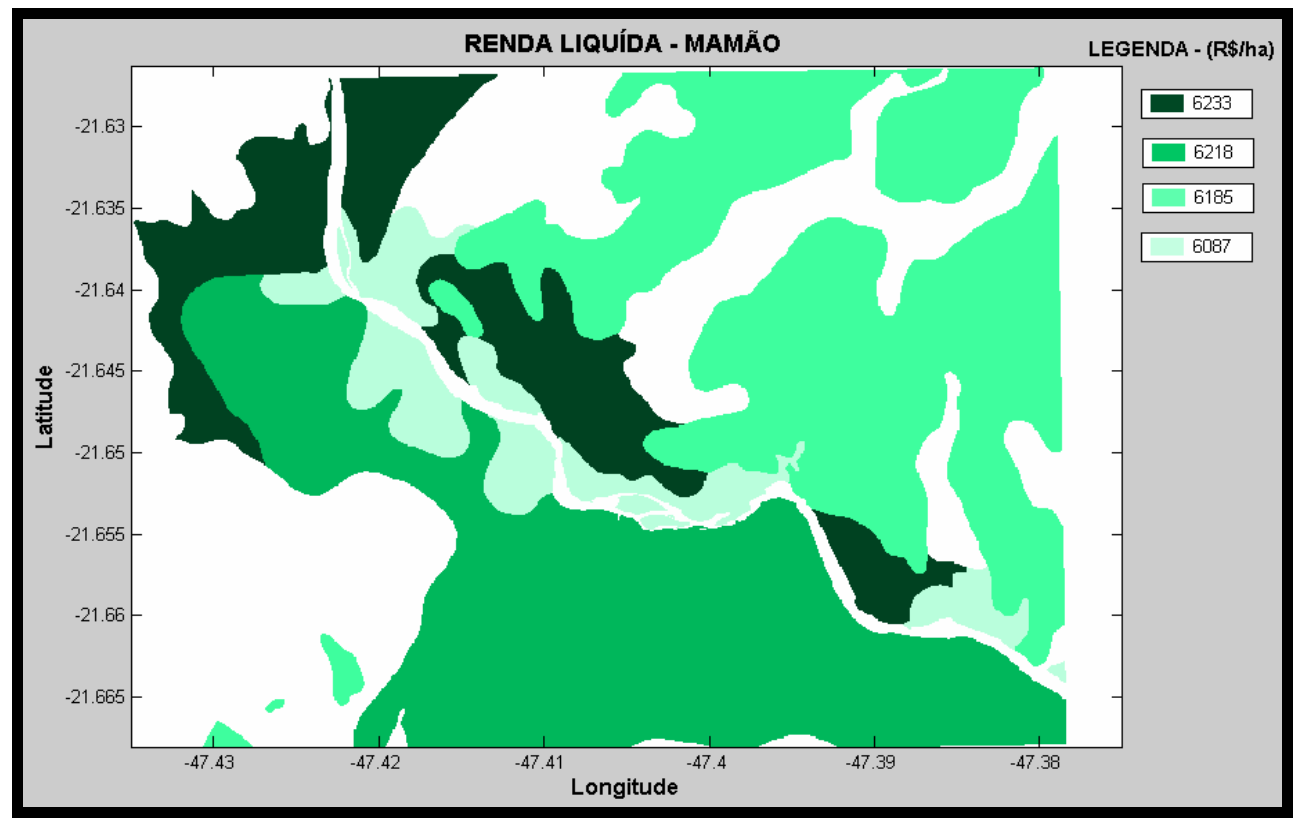

FIGURA 6. Tela de saída da rotina computacional, mostrando a renda líquida da cultura do mamão. Output screen of computational routine showing the net income of papaya culture.

Os resultados mostraram variação nos valores de renda das culturas nos diferentes tipos de solo da área estudada. Com exceção da cultura do abacaxi, os maiores valores ocorreram na classe de solo representado pela unidade de mapeamento $\mathrm{Ce} 4$, em função das melhores características físicas e químicas que essa classe de solo apresenta, comparada com as demais.

Também foi possível notar que, com exceção da cultura da cana-de-açúcar, o menor valor de renda ocorreu na classe de solo representada pela unidade de mapeamento Aed3, devido a sua menor fertilidade natural e maior acidez, comparada com as demais classes de solo, exigindo maiores gastos com adubação e calagem.

De modo geral, as classes de solos que indicaram menores desembolsos com correção de acidez e de adubação complementar, como, por exemplo, a classe representada pela unidade de 
mapeamento $\mathrm{Ce} 4$, ou seja, as de melhores fertilidade natural e características físicas, apresentaram maior renda líquida. Por outro lado, quando a classe apresentava baixa fertilidade natural, combinada com características físicas que contribuem para baixa retenção de água no solo, como exemplo a classe representada pela unidade de mapeamento Aed3, apresentaram menor renda líquida.

Uma análise de sensibilidade realizada para a cultura da cana-de-açúcar mostrou que só seria viável o seu plantio se o preço da tonelada por hectare fosse de, no mínimo, $\mathrm{R} \$ 56,00$. Quando foi adotado o preço de 24,5 $\mathrm{R} \$ \mathrm{t}^{-1}$, recomendado pelo AGRIANUAL (2004), o algoritmo sugeriu não plantar essa cultura, considerando o cenário da área em estudo.

A variação na renda líquida foi mais influenciada pelas características dos diferentes tipos de solos do que pelo custo da água, devido ao baixo custo da água para irrigação adotada pelo CEIVAP. Os resultados apresentados na Tabela 4 e nas Figuras de 3 a 6 possibilitam escolher a melhor opção de plantio de forma a se obterem as melhores rendas na área estudada, mostrando que a metodologia aqui adotada pode ser uma ferramenta útil no planejamento da agricultura irrigada. Procedimento semelhante pode ser implementado para outras culturas, desde que sejam conhecidas suas funções de resposta à água.

As culturas do mamão e do abacaxi apresentaram os melhores desempenhos, comparadas com as demais, em função do menor custo de produção, associado à maior eficiência no uso da água e aos melhores preços de venda da produção.

\section{CONCLUSÕES}

O algoritmo computacional desenvolvido mostrou-se eficiente para o mapeamento da renda líquida das culturas, podendo ser utilizado no planejamento da agricultura irrigada para outras culturas e regiões.

O cenário agrícola que apresentou os maiores retornos econômicos, com base no algoritmo computacional desenvolvido, sugere o cultivo das culturas do abacaxi e do mamão na região norte fluminense.

\section{REFERÊNCIAS}

AGRIANUAL: anuário da agricultura brasileira. São Paulo: FNP Consultoria e Comércio, 2004. $546 \mathrm{p}$.

ALMEIDA, F.T. Respostas do mamoeiro 'Improved Sunrise Solo 72/12' a diferentes lâminas de irrigação no Norte Fluminense. 2000. 128 f. Tese (Doutorado em Produção Vegetal) - Universidade Estadual do Norte Fluminense, Campos dos Goytacazes, 2000.

ASSAD, E.D.; SANO, E.E. Sistemas de informações geográficas (Aplicações na Agricultura). 2.ed. Brasília: SPI/EMBRAPA-CPAC, 1998. 434 p.

CÂMARA, G.; MEDEIROS, J.S. Princípios básicos em geoprocessamento. In: ASSAD, E.D.; SANO, E.E. Sistemas de Informações Geográficas (Aplicações na Agricultura). 2.ed. Brasília: SPI/ EMBRAPA-CPAC, 1998. 434 p.

CARVALHO, D.F.; BONOMO, R.; GRIEBELER, N.; RIBEIRO, A. Estimativa da demanda máxima de irrigação e da duração do ciclo para a cultura do milho (Zea mays L.), na bacia do rio Verde Grande (MG). Ciência e Agrotecnologia, Lavras, v.22, n.1, p.97-104, 1998.

CARVALHO, D.F.; CRUZ, E.S.; MOREIRA, J.F. Disponibilidade climática no Estado do Rio de Janeiro, visando à elaboração de projetos de irrigação. In: Congresso Brasileiro de Engenharia Agrícola, 30., 2001, Foz do Iguaçu. Anais... Cascavel: SBEA, 2001. 1 CD-ROM. 
CARVALHO, D.F.; SILVA, W.A.; CEDIA M.B.; TANAJURA, E.L.X.; VILLELA A.L.O.

Estimativa do custo de implantação da agricultura irrigada, utilizando o sistema de informação geográfica. Engenharia Agrícola, Jaboticabal, v.25, n.2, p.395-408, 2005.

CARVALHO, D.F.; MOREIRA, J.F.; CRUZ, E.S; SILVA, W.A.; OLIVEIRA, L.F.C. Comparação entre diferentes metodologias de estimativa da evapotranspiração de referência e sua influência na demanda máxima de irrigação para o milho (Zea mays L.) no Estado do Rio de Janeiro. Revista Agronomia, Seropédica, v.36, n.1/2, p.48-55, 2002.

CEDDIA, M.B. Zoneamento agroambiental e indicadores de sustentabilidade como subsídio ao planejamento agrícola do municícpio de Paty do Alferes - RJ. 2000. 297 f. Tese (Doutorado) Universidade Federal Rural do Rio de Janeiro, Seropédica, 2000.

CEIVAP, 2003. Cobrança pelo uso da água na Bacia do Rio Paraíba do Sul. Disponível em: $<$ http://www.ceivap.org.br./ceivap_1.php>. Acesso em: 17 ago. 2006.

DE-POLLI, H. Manual de adubação para o Estado do Rio de Janeiro. Itaguaí: UFRRJ, 1988. 179 p. (Coleção UFRRJ: Ciências Agrárias, 2).

FARIA, R.A.; SOARES, A.A.; SEDIYAMA, G.C.; RIBEIRO, C.A.A. Economia de água e energia em projetos de irrigação suplementar no Estado de Minas Gerais. Revista Brasileira de Engenharia Agrícola e Ambiental, Campina Grande, v.6, n.2, p.189-94, 2002.

JUSSIANE, L.F. Desempenho do método de Lagrangiano Aumentado com penalidade quadrática. 2004. 108 f. Dissertação (Mestrado em Matemática) - Universidade Federal do Paraná, Curitiba, 2004.

MARTINS, D.P. Resposta do maracujazeiro-amarelo (Passiflora edulis Sins var. flavicarpa) $a$ lâminas de irrigação e dose de nitrogênio e potássio. 1998. 84 f. Tese (Doutorado em Produção Vegetal) - Universidade Estadual do Norte Fluminense, Campos dos Goytacazes, 1998.

REGO FILHO, L.M. Respostas do Abacaxizeiro "Pérola" a diferentes lâminas de irrigação no norte Fluminense. 2002. 150 f. Tese (Doutorado em Produção Vegetal) - Universidade Estadual do Norte Fluminense, Campos dos Goytacazes, 2002.

RODRIGUEZ, J.A.; LOPEZ, G. Planificación de recursos para la modernización de los sistemas arroceros mediante el empleo de modelos de simulación y SIG. Investigacción Agraria: Producción y Protección Vegetales, Havana, v.15, n.3, p.181-94, 2000.

SILVA, W.A. Planejamento de áreas irrigadas em assentamentos rurais utilizando os Sistemas de Informações Geográficas. 2003. 83 f. Dissertação (Mestrado em Fitotecnia) - Universidade Federal Rural do Rio de Janeiro, Seropédica, 2003.

SILVA, W.A. Modelagem matemática aplicada no planejamento da agricultura irrigada, utilizando informações georreferenciadas. 2007.98 f. Tese (Doutorado em Ciência do Solo) Universidade Federal Rural do Rio de Janeiro, Seropédica, 2007.

SILVA, W.A.; CARVALHO, D.F.; CEDIA M.B.; TANAJURA, E.L.X.; VILLELA, A.L.O. Estimativa do custo de implantação da agricultura irrigada, utilizando o sistema de informação geográfica. Engenharia Agrícola, Jaboticabal, v.25, n.2, p.395-408, 2005.

SOUSA, E. F. Função de produção da cana-de-açúcar e da goiabeira em relação à irrigação. 1997. 119 f. Tese (Doutorado em Produção Vegetal) - Universidade Estadual do Norte Fluminense, Campos dos Goytacazes, 1997. 